Classification Physics Abstracts 29.40

\title{
MULTIPLICATEUR A GALETTE DE MICROCANAUX : AMÉLIORATION DES PERFORMANCES DE GAIN ET DE DYNAMIQUE DE DÉTECTION
}

\author{
M. AUdier, J. C. DELMOTTE et J. P. BOUTOT \\ Laboratoires d'Electronique et de Physique Appliquée, \\ 3, avenue Descartes, 94450 Limeil Brevannes, France
}

(Reçu le 18 juillet 1977, révisé le 19 décembre 1977, accepté le 23 décembre 1977)

\begin{abstract}
Résumé. - Les performances d'une GMC dépendent directement de ses caractéristiques géométriques (diamètre $d$ et rapport $1 / d$ des canaux, surface utile) et de la tension appliquée à ses bornes. Les performances de gain et de débit sont limitées par des phénomènes de saturation.

Une association particulière de deux GMC montées en cascade permet d'améliorer simultanément les caractéristiques de gain, fluctuations de gain et dynamique de détection (niveau détecté, taux de comptage). Il est ainsi possible d'obtenir, pour des gains $10^{6}<G<\mathrm{qq} \mathrm{107}$, une dispersion $\Delta G / \bar{G} \leqslant 20 \%$ et d'améliorer la dynamique de détection d'un facteur $>10$.

Abstract. - The performances of an MCP are a function of its geometrical characteristics (diameter $d$ and ratio $1 / d$ of a channel, useful area) and of the applied voltage. Gain and mean output current are limited by saturation phenomena.

By using a particular cascaded MCP's configuration, it is possible to simultaneously improve the gain, its associated fluctuations and the detection dynamics (detected level, counting rate). For gains $10^{6}<G<$ a few $10^{7}$, the fluctuations can be kept as low as $20 \%$ and an improvement by a factor $>10$ can be obtained on the detection dynamics.
\end{abstract}

1. Introduction. - Dans bien des expériences, notamment en Physique des Hautes Energies, le détecteur à scintillations s'impose du fait de ses performances élevées de sensibilité, linéarité et rapidité [1].

Dans certaines conditions expérimentales, la détection peut être réalisée également par une conversion directe des événements (ions, UV, X par exemple) en électrons (PM sans fenêtre).

Les limites de performances d'un PM sont liées, pour une bonne part, à la conception même de la structure multiplicatrice à cascade de dynodes. Plusieurs types de structures sont couramment utilisées (structure linéaire focalisée, à persiennes...) ; elles se différencient par leurs caractéristiques de rapidité, linéarité, stabilité, etc... [2].

Le multiplicateur d'électrons à galette de microcanaux (GMC), étudié à l'origine pour l'amplification d'images électroniques à bas niveau, offre, de par sa conception particulière et ses caractéristiques géométriques, des possibilités nouvelles pour la conception de détecteurs plus performants ou originaux [3].

Comme nous le verrons, les performances des GMC varient notablement avec les dimensions géométriques des canaux (diamètre $d$, longueur $l$ ) ainsi qu'avec leur surface utile $S$. Pour fixer une idée, $d$ est généralement de l'ordre d'une dizaine à quelques dizaines de microns
( $d$ typiques : $12,5,25,40$ et $100 \mu \mathrm{m}$ ) ; les surfaces, en principe circulaires et communément de quelques $\mathrm{cm}^{2}$, peuvent atteindre une centaine de $\mathrm{cm}^{2}$. Un rapport $1 / d$ de quelques dizaines (40 à 100) étant généralement nécessaire, l'épaisseur d'une GMC peut varier de quelques $1 / 10^{\mathrm{e}} \mathrm{mm}$ à quelques $\mathrm{mm}$.

Les tensions $V_{\mathrm{G}}$ appliquées aux bornes des GMC sont du même ordre que celles utilisées pour les multiplicateurs conventionnels mais, compte tenu des très faibles dimensions, ies champs électriques qui en résultent sont très élevés (quelques $100 \mathrm{~V} \cdot \mathrm{mm}^{-1}$ à plus de $1 \mathrm{kV} . \mathrm{mm}^{-1}$ ).

Une des limitations dans les applications des GMC à la réalisation de $\mathrm{PM}$ a été le gain, limité, en pratique, à quelques $10^{4}$ à cause des réactions ioniques favorisées par la géométrie rectiligne des canaux. Il a été montré que la limite de gain d'une GMC peut être atteinte si les canaux sont courbés (réduction importante des effets dus aux ions). Pour une GMC, le gain et le produit gain-dynamique de détection sont limités et fixés par ses caractéristiques géométriques et électriques. Toutefois, on montre qu'une amélioration simultanée des caractéristiques de gain, fluctuations de gain et dynamique de détection peut être obtenue par une association particulière de deux GMC montées en cascade. 
2. Rappel des caractéristiques générales des GMC. Galettes à canaux courbes (GMC). - 2.1 CARACTÉRISTIQUES DE GAIN. - Il est bien connu que le processus de multiplication dans un canal est limité par trois phénomènes : l'ionisation des gaz, la saturation en charge et en courant, liées à la fois à la géométrie et à la nature même de la dynode continue.

2.1.1 Réactions ioniques. - De par son principe de fonctionnement, le gain d'un canal est une fonction assez complexe de la tension appliquée à ses bornes, de son diamètre $d$ et du rapport $1 / d$. Des gains supérieurs à $10^{5}$ peuvent être obtenus si le rapport $1 / d$ est suffisamment grand [4]. En pratique, de tels gains ne sont guère utilisables avec des canaux droits car, à ces niveaux, les ions créés à l'intérieur des canaux (formation quasi inévitable du fait de la présence de gaz résiduels et de gaz adsorbés sur les parois) sont la source de phénomènes parasites par réaction avec l'entrée des canaux (post-impulsions, voire autoentretiens). On sait que la mise en proximité d'une surface émissive (telle qu'une photocathode) avec l'entrée d'une GMC favorise grandement ces phénomènes.

Parmi les solutions étudiées afin de limiter les retours d'ions il faut citer la mise en cascade de deux GMC à canaux droits (GMCD) suivant une configuration particulière dite en chevron (Fig. 1a). Un tel montage permet de plus, comme nous l'expliquerons par la suite, d'accroître notablement le gain d'un multiplicateur à GMC.
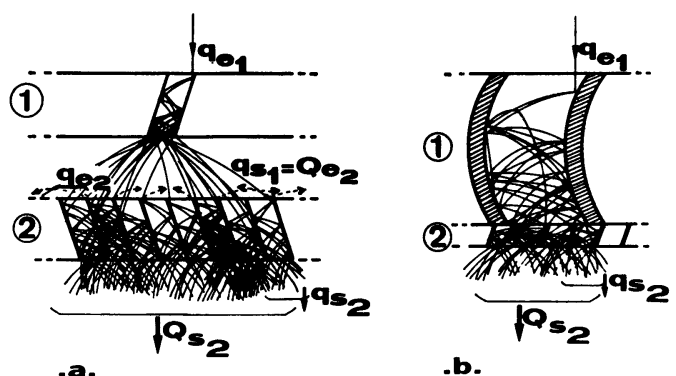

FIG. 1. - Association en cascade de deux GMC. Principe de fonctionnement : a) montage chevron de GMC à canaux droits; b) association GMCC-GMCD avec couplage par contact.

[Cascaded MCP. Operation principle : a) chevron association of two straight channel MCP ; b) GMCC-GMCD association with direct coupling.]

Une solution permettant de faire fonctionner convenablement une seule GMC à des gains élevés $\left(G>10^{5}\right)$ - et qui s'est montrée très efficace dans le cas des multiplicateurs monocanaux - consiste à courber les canaux de la GMC : cette solution a pu être mise en œuvre grâce à une technologie étudiée à LEP (Fig. 2). On a montré qu'il est ainsi possible d'accroître le gain jusqu'au niveau correspondant à la saturation quasi complète de l'impulsion de charge en sortie du canal sans que les retours d'ions viennent perturber le mécanisme de multiplication [5].

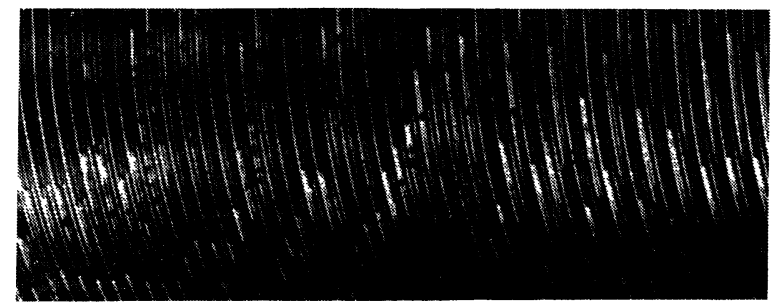

FIG. 2. - Coupe d'une GMC à canaux courbes.

[Cross-section view of a curved channel MCP.]

\subsubsection{Fluctuations statistiques de gain. Saturation en} charge. - Lorsqu'une GMC fonctionne en régime linéaire (impulsion de charge par canal non saturée), la distribution du gain en électron unique ou spectre d'électron unique (SEU) est représentée assez bien par une loi exponentielle négative, ce qui donne une variance relative de gain $V_{\mathrm{r}}=\sigma^{2} / G^{2}=1$ (où $\sigma$ représente l'écart type des fluctuations) (Fig. 3, courbe $a$ ).

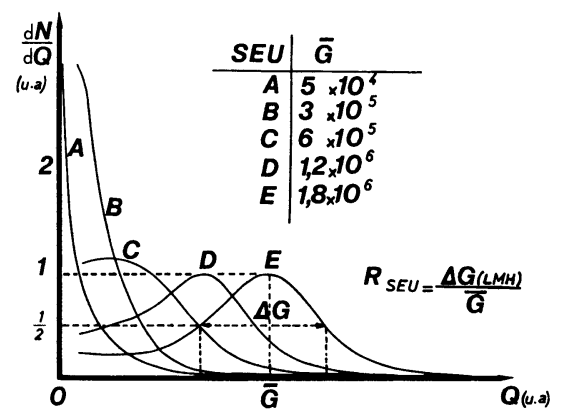

Fig. 3. - Evolution du spectre d'électron unique d'une GMCC. [Single electron spectrum evolution of a curved channel MCP.]

Cependant, un effet de saturation en charge dans l'avalanche électronique apparaît lorsque la quantité de charges en sortie atteint un certain niveau : le gain d'un canal ne croît plus alors aussi rapidement avec la tension appliquée $V_{\mathrm{G}}$. Le seuil de saturation d'un microcanal $G_{0 \text { sat. }}$. est une fonction de son diamètre $d$. Les gains saturés typiques que l'on peut ainsi atteindre sont de quelques $10^{5}$ pour $d=12,5 \mu \mathrm{m}$ à quelques $10^{6}$ pour $d=40 \mu \mathrm{m}$.

Le processus de saturation a pour effet immédiat de réduire la fluctuation du gain $G$. Cette évolution est progressive à partir du gain $G_{0 \text { sat. }}$ (Fig. 4) et, comme le montre la figure 3 (courbe $e$ ), la distribution du SEU devient quasi gaussienne au gain saturé $\left(G \simeq 10^{6}\right)$. Une valeur typique de résolution

$$
R_{\mathrm{SEU}}=\frac{\Delta G(\mathrm{LMH})\left(^{*}\right)}{\bar{G}} \simeq 50 \%
$$

peut ainsi être obtenue avec une seule GMCC.

(*) LMH = largeur à mi-hauteur. 


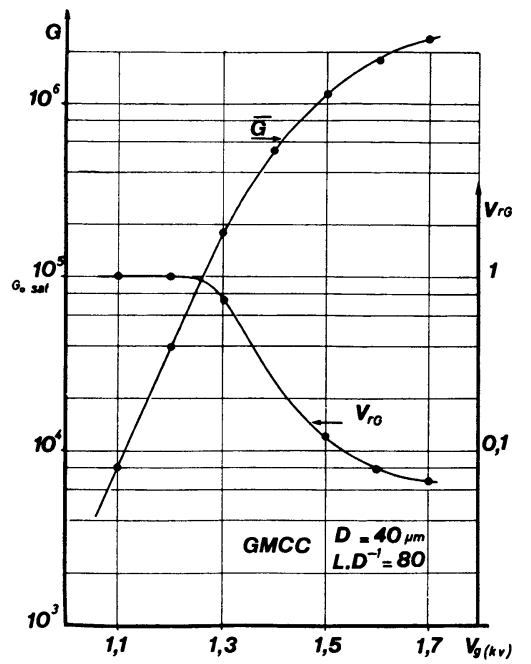

Fig. 4. - Courbe de gain et variance relative de gain d'une GMCC.

[Gain mean value and gain relative variance of a GMCC.]

2.2 Linéarité De RéPonse. DyNAMiQue de DÉTeCTION D'UNE GMC. - En régime continu, le courant de sortie $I_{\mathrm{S}}$ évolue de façon non linéaire en fonction du courant d'entrée $I_{\mathrm{E}}\left(I_{\mathrm{S}}<G . I_{\mathrm{E}}\right)$ dès qu'il approche une fraction $k\left(10^{-2}<k<10^{-1}\right)$ du courant de conduction $I_{\mathrm{C}}=V_{\mathrm{G}} / R_{\mathrm{G}}$ de la galette $\left(R_{\mathrm{G}}=\right.$ résistance de la galette). On a donc la condition de linéarité : $I_{\mathrm{S}}<k \cdot I_{\mathrm{C}}$.

$\mathrm{Si}$ nous considérons des galettes de matériau de même résistivité avec des rapports $L . d^{-1}$ identiques, $R_{\mathrm{G}}$ varie comme $d^{2} / S\left(R_{\mathrm{G}}=\rho \frac{L . d^{-1}}{N \pi}, \rho\right.$ étant la résistivité du matériau, $N=a \frac{S}{d^{2}}$ le nombre de microcanaux, $S$ la surface de la galette et $a$ le facteur d'ouverture). On voit alors que la dynamique en courant par unité de surface est d'autant plus élevée que le diamètre des canaux est petit (ce qui, notons-le, va à l'encontre d'un gain élevé).

- En régime impulsionnel, la notion de linéarité de réponse est plus complexe pour une GMC que pour un multiplicateur conventionnel du fait de la multiplicité $(N)$ des multiplicateurs (canaux) susceptiblcs de fonctionner simultanément et de la haute résistivité du matériau (qq $10^{12} \Omega / \square$ ). Cette dernière caractéristique rend le temps de recharge d'un canal (temps de récupération) extrêmement long devant la durée des impulsions généralement considérées : ce temps de recharge $\tau_{\mathrm{r}}$ est le temps nécessaire pour que la charge positive créée sur la paroi d'un canal, lorsqu'une charge q a été délivrée, puisse être annulée par le courant de conduction $i_{\mathrm{c}}$ de ce même canal $\left(i_{\mathrm{c}}=I_{\mathrm{C}} / N\right)$. La conductivité du microcanal étant beaucoup plus élevée en volume qu'en surface [6] (au moins deux fois plus élevée à $10 \mathrm{~nm}$ de la surface), il existe une liaison résistive et capacitive entre chaque palier de multiplication et le pont de conduction qui impose une constante de temps $\tau_{\mathbf{r}}$ au recouvrement des charges positives par le courant $i_{\mathrm{c}}$.

Les valeurs typiques de courant $i_{\mathrm{c}}$ étant de quelques $10^{-11} \mathrm{~A}$, on voit que pour une charge délivrée de $10^{6} . \bar{e}$, le temps de recharge $\tau_{\mathrm{r}}$ est supérieur à $10^{-3} \mathrm{~s}$.

Pour définir la linéarité de réponse d'une GMC, cette notion de temps mort nécessite de prendre en considération la récurrence $F$ des impulsions. On ne considère ici que le cas d'impulsions brèves devant $\tau_{\mathrm{r}}$.

Dans le cas de la détection d'impulsions ( $n$ électrons) isolées, c'est-à-dire suffiamment espacées dans le temps $\left(F<1 / \tau_{\mathrm{r}}\right)$, la linéarité de réponse en charge est conservée, quel que soit le gain $\bar{G}_{0}$, tant que la charge delivrée $\bar{Q}_{\mathrm{S}_{0}}=\bar{n} . e^{-} \cdot \bar{G}_{0}$ ne dépasse pas une certaine valeur $N \times \bar{q}_{\mathrm{s}_{0}}$ où $\bar{q}_{\mathrm{s}_{0}}$ représente la charge maximale ramenée à un canal que peut fournir linéairement une GMC comportant $N$ canaux utiles [7].

La figure 5 (courbes $b$ ) montre l'évolution du gain moyen $\bar{G}_{0}$ d'un multiplicateur en fonction de la quantité de charges, ramenée à un microcanal, qu'il détecte $\left(\bar{q}_{\mathrm{e}}=\bar{Q}_{\mathrm{S}} / \bar{G}\right)$.

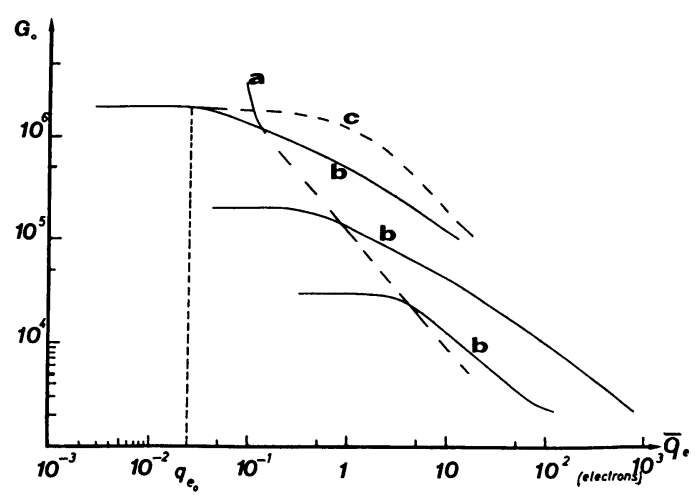

Fig. 5. - Linéarité de détection d'une $\operatorname{GMC}(d=40 \mu \mathrm{m})$ : a) limite théorique de la dynamique de détection en électrons par microcanal d'une GMC; $b$ ) évolution du gain moyen $\bar{G}_{0}$ en fonction de la charge moyenne $\bar{q}_{\mathrm{e}}$ détectée par microcanal ; c) évolution théorique du gain en fonction de l'empilement de plusieurs événements primaires par canal, en régime de gain saturé.

[MCP response linearity.]

A faible gain $\left(\bar{G}_{0} \ll \bar{G}_{\text {sat. }}\right)$, la valeur $\bar{q}_{\mathrm{e}_{0}}$, correspondant au niveau maximum de détection en régime linéaire $\left(\bar{G}=\bar{G}_{0}\right)$, répond assez bien à la charge calculée en considérant la GMC comme un simple condensateur :

$$
\bar{Q}_{\mathrm{S}_{0}}=\varepsilon S\left(E_{(0)}-E_{(\mathrm{L})}\right)
$$

soit

$$
\bar{q}_{\mathrm{e}_{0}}=\frac{\bar{Q}_{\mathrm{s}_{0}}}{N \bar{G}_{0}}=\frac{\varepsilon S}{N \bar{G}_{0}}\left(E_{(0)}-E_{(\mathrm{L})}\right)
$$

où $\varepsilon$ est la constante diélectrique moyenne du milieu, $S / N$ la surface de galette occupée par microcanal, $E_{(0)}$ et $E_{(\mathrm{L})}$ les champs électriques respectivement à l'entrée et à la sortie des canaux. Lorsque la saturation 
apparaît, on considère que $E_{(\mathrm{L})}$ est alors égal à la valeur $E_{1}$ conduisant à un gain moyen de 1 par unité de longueur $\left(E_{1} d \# 9 V\right)$ [12] tandis que $E_{(0)}$ est pratiquement égal à la valeur initiale $E_{0}$ du champ dans le canal. L'expression de $\bar{q}_{\mathrm{e}_{\mathrm{o}}}$ 'écrit alors (Fig. 5, courbe $a$ )

$$
\bar{q}_{\mathrm{e}_{0}} \# \frac{4 \times 10^{8} d}{\bar{G}_{0}}\left[\frac{V_{\mathrm{G}}}{L \cdot d^{-1}}-9\right] \quad(\mathrm{MKS}) !
$$

Pour des gains $\bar{G}_{0}$ très élevés $\left(\bar{G}_{0}>G_{0 \text { sat }}\right)$, chaque microcanal étant saturé par la multiplication d'un seul événement, la linéarité de détection se trouve limitée d'une part, à l'entrée, par l'empilement de plusieurs événements par microcanal (voir Annexe 1 et Fig. 5, courbe $c$ ) et, d'autre part, lors de la multiplication, par la modification de la distribution des équipotentielles due à un couplage électrostatique entre canaux voisins. Une certaine linéarité de fonctionnement peut néanmoins être conservée lorsque, lors de la détection d'un signal de répartition uniforme sur l'entrée du multiplicateur, le rapport nombre d'électrons détectés (n) au nombre de microcanaux disponibles $(N)$ est suffisamment faible (défaut de linéarité $\leqslant 1 \%$ pour $\bar{n} / N \leqslant 1 / 100)$.

- Considérons maintenant le cas de la détection d'impulsions répétitives dont le taux $F$ est tel que $F>1 / \tau_{\mathrm{r}}$ : en plus de la condition $\bar{Q}_{\mathrm{s}}<N \cdot \bar{q}_{\mathrm{s}}$, la linéarité de réponse ne pourra être préservée que si le courant moyen de sortie $\bar{I}_{\mathrm{S}}$ donné par $\bar{I}_{\mathrm{S}}=F \cdot \bar{n} \cdot e^{-} . \bar{G}$ reste dans le domaine de réponse linéaire en régime continu, soit $F \cdot \bar{n} \cdot e^{-} \cdot \bar{G}<k \cdot N \cdot \bar{i}_{\mathrm{c}}$. Ainsi, dans le cas de la détection d'événements uniques $(\bar{n}=1)$ pour une galette de canaux $d=40 \mu \mathrm{m}$, de résistance $R_{\mathrm{G}}=10^{9} \Omega \mathrm{cm}^{-2}$, le taux de comptage maximum au gain saturé de $10^{6}\left(V_{\mathrm{G}}=1500 \mathrm{~V}\right)$ est :

$$
F_{\mathrm{M}} \simeq k \times 10^{7} \mathrm{~s}^{-1} \cdot \mathrm{cm}^{-2}
$$

soit $F_{\mathrm{M}} \simeq 10^{5}$ à $10^{6} \mathrm{imp} \cdot \mathrm{s}^{-1} \cdot \mathrm{cm}^{-2}$.

3. Multiplicateur à cascade de GMC. 3.1 GALETTES EN CHEVRON. - Une structure multiplicatrice associant en cascade 2 GMCD juxtaposées de telle sorte que les canaux soient orientés suivant une configuration de chevron a déjà été proposée afin de limiter les réactions ioniques [8]. W. Parkes et R. Gott [9], W. B. Colson et al. [10] ont montré qu'une telle association permettait d'atteindre des gains égaux ou supérieurs à $10^{7}$ avec une distribution quasi gaussienne de résolution $R_{\mathrm{SEU}}>100 \%$. De meilleures résolutions, de l'ordre de $50 \%$, peuvent être obtenues par l'utilisation de GMC de grand rapport $1 / d$ $(\geqslant 80)[11]$.

Ces gains importants, aux fluctuations réduites, sont obtenus en faisant débiter, pour chaque événement détecté par canal de la première galette, $N$ canaux de la seconde (Fig. 1a). Généralement, on limite à quelques $10^{3}$ le coefficient de multiplication moyen du premier étage afin de rendre négligeable la probabilité de création d'ions au niveau des derniers paliers de multiplication.

Dans ces conditions, les impulsions de charges débitées par la première galette $\left(q_{\mathrm{s}_{1}}\right)$ ont une amplitude qui suit une loi de probabilité quasi exponentielle décroissante. $\mathrm{Si} N$ n'est pas trop grand et si chaque canal reçoit suffisamment d'électrons, il se produit, après multiplication, une saturation en charge conduisant à un fonctionnement en régime de canal saturé. La fluctuation de la quantité de charges délivrées par chacun de ces $N$ canaux $\left(q_{\mathrm{s}_{2}}\right)$ se trouve alors réduite et suit une loi de distribution quasi gaussienne. Dans cette association, les ions susceptibles d'être produits en grand nombre en sortie des canaux de la seconde GMC sont stoppés par impact sur la face de sortie de la première GMC.

Pour chaque événement détecté, le signal électronique global, donc le gain, obtenu à partir d'une telle association, est donné par la somme $Q_{\mathrm{s}_{2}}$ des charges $q_{\mathrm{s}_{2}}$ délivrées par les $N$ canaux de la seconde GMC :

$$
Q_{\mathrm{s}_{2}}=\sum_{i=1}^{N} q_{\mathrm{s}_{2 i}} \text {. }
$$

Des valeurs de gains élevées $\left(10^{7}\right.$ à $\left.10^{8}\right)$ peuvent être obtenues : elles dépendent essentiellement du type de canaux utilisés et de la défocalisation du faisceau d'électrons dans l'espace inter-galettes (variation sur $\bar{N}$ ) (Fig. 1a).

La fluctuation en amplitude du signal $Q_{\mathrm{S}_{2}}$ dépend donc d'une part des fluctuations de $N$ et, d'autre part, de celles de $q_{\mathrm{s}_{2}}$. En supposant que les $N$ canaux travaillent de manière identique (gain et charge détectée $q_{\mathrm{e}_{2}}$ ), l'amplitude $\bar{Q}_{\mathrm{s}_{2}}$ du signal obtenu est donnée par : $\bar{Q}_{\mathrm{s}_{2}}=\bar{N} \cdot \bar{q}_{\mathrm{s}_{2}}$, dont la variance relative $V_{\mathrm{r}_{\mathrm{s}_{\mathrm{s}}}}$ est :

$$
V_{\mathrm{r}_{\mathrm{Qs}}}=V_{N}+\frac{V_{q_{\mathrm{s} 2}}}{N}
$$

En réalité, pour un couplage lâche (galettes espacées, $\bar{N}$ grand), la charge détectée $\left(q_{\mathrm{e}_{2}}\right)$ (Fig. $\left.1 a\right)$ n'est pas la même sur chacun des $\mathrm{N}$ canaux (distribution angulaire de la charge $q_{\mathrm{s}_{1}}$ en sortie de la première GMC non uniforme). La charge $q_{\mathrm{s}_{2}}$ ne sera la même pour les $N$ canaux que si la galette fonctionne en régime de gain saturé. Dans ces conditions, il est possible d'obtenir des résolutions en amplitude $80 \%<R_{\mathrm{SEU}}<100 \%$. Cependant, un fonctionnement à gain élevé de la seconde galette n'est pas favorable à l'obtention d'un faible bruit (post-impulsions saturées).

Dans le cas d'un chevron, la fluctuation de la charge délivrée par la première $\operatorname{GMC}\left(q_{\mathrm{s}_{0}}\right)$, cause de la fluctuation de $q_{\mathrm{e}_{2}}$ sur chacun des $N$ canaux, entraîne, pour la seconde GMC, un régime de fonctionnement qui peut correspondre à un large domaine de sa caractéristique de linéarité, recouvrant à la fois les zones de fonctionnement linéaire et saturé. Pour que chacun des $N$ canaux travaille en saturation, il est nécessaire que $q_{\mathrm{e}_{2}}$ soit toujours supérieur à $q_{\mathrm{e}_{0}}$, c'est-àdire que la première GMC travaille à gain élevé et à 
fluctuations de gain réduites (Fig. 6). Cette condition peut être remplie en utilisant, comme premier étage, une GMC de grand $1 / d$ fonctionnant en régime de gain saturé. C'est selon ce principe qu'ont pu être obtenues

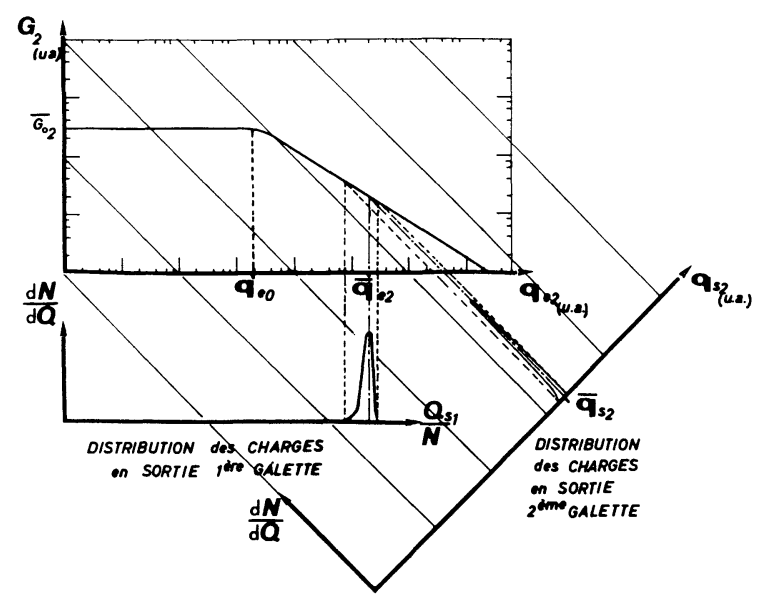

Fig. 6. - Distribution des charges en sortie de la première galette GMCC et de la seconde galette GMCD. Réduction des fluctuations de gain.

[Charge distribution at the output of the 1st and the 2nd MCP. Reduction of gain fluctuations.]

des résolutions $R_{\mathrm{SEU}} \simeq 50 \%$ à l'aide de chevrons à canaux droits. Cependant, la protection contre les retours ioniques n'est plus assurée, d'où l'intérêt de l'utilisation d'une GMCC.

Comme nous l'avons vu, un couplage lâche permet d'accroître le gain, mais, en contre partie, introduit simultanément une variation du niveau de détection par canal et des fluctuations sur $N$ (effet de moiré, épanouissement du faisceau par effets secondaires). Un couplage par contact des galettes permet, d'une part, d'uniformiser la répartition des charges sur les $N$ canaux et, d'autre part, de supprimer les fluctuations liées à l'épanouissement du faisceau, ce qui ne peut être que partiellement obtenu avec une focalisation de proximité.

Pour des canaux de même diamètre $d$, un couplage par contact donne une valeur faible pour $N$ $(1 \leqslant N \leqslant 3)$. $V_{N}$ peut être réduit en associant aux canaux du premier étage des canaux de plus petit diamètre $\left(d_{2}<d_{1}\right)$ (Fig. $\left.1 b\right)$.

Afin d'obtenir un faible taux de post-impulsions (rapport en amplitude impulsion parasite/impulsion de signal), il est nécessaire de faire fonctionner la seconde GMC à faible gain : ceci est rendu possible par la valeur élevée de $q_{\mathrm{e}_{2}}$ (Fig. 5 et 6). Cependant, pour obtenir une charge de sortie $q_{\mathrm{s}_{2}}$ élevée, il faut maintenir $V_{G_{2}}$ suffisamment grand (cf. expression de $q_{\mathrm{e}_{0}}$ sachant que $q_{\mathrm{s}_{2}}>G_{0} q_{\mathrm{e}_{0}}$. Il est alors nécessaire, comme le montrent les courbes universelles de gain [12], de choisir, pour cette seconde GMC, un rapport $1 / d$ faible.

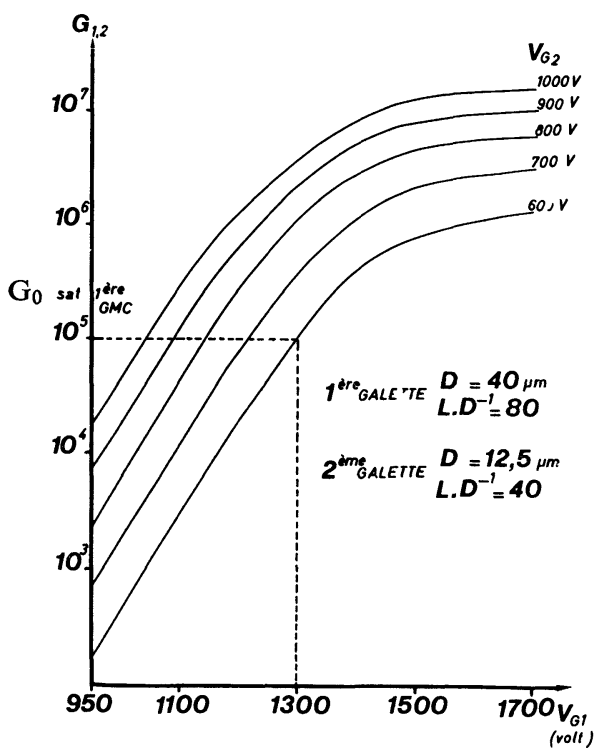

Fig. 7. - Caractéristiques de gain d'une cascade GMCCGMCD.

[Gain curves of a GMCC-GMCD association.]

3.2 Résultats eXPéRIMENTAUX. - Des mesures ont été effectuées sur un multiplicateur répondant aux définitions précédemment exposées :

- un premier étage à GMCC $\left(d_{1}=40 \mu \mathrm{m}\right.$, $L_{1} d_{1}^{-1}=80$ ) dont le fonctionnement (gain, dynamique de détection) est tel qu'il a été décrit précédemment (cf. § 2.2);

- un second étage à GMCD $\left(d_{2}=12,5 \mu \mathrm{m}\right.$ $L_{2} d_{2}^{-1}=40$ ) qui, compte tenu de la précédente multiplication, fonctionne en régime de saturation complète $\left(q_{\mathrm{e}_{2}} \gg q_{\mathrm{e}_{20}}\right)$ et se comporte en régulateur de gain (son coefficient de multiplication $G_{2}$ étant quasi inversement proportionnel au niveau de charge détecté)

- couplage par contact.

3.2.1 Caractéristique de gain et linéarité de détection. - Le réseau de courbes (Fig. 7) montre que l'effet de saturation sur la caractéristique de gain est plus marqué que celui obtenu avec une seule GMCC (Fig. 4). En régime de saturation, le gain $G$ est ajustable à quelques $10^{7}$ par la tension appliquée sur le second étage.

L'étude des fluctuations de gain montre que le rapport signal sur bruit $\left(S / B=V_{\mathrm{r}_{\mathrm{Q}}}^{-1 / 2}\right)$, considérablement amélioré par le fonctionnement en saturation de charge de la seconde galette, est directement proportionnel à celui de la GMCC.

Les différents montages étudiés ont donné des résolutions $6 \% \leqslant R_{\mathrm{SEU}} \leqslant 20 \%$, soit $12<S / B<23$ (Fig. 8). Cette résolution est quasi indépendante de la tension appliquée sur la seconde GMC.

Cette réduction de fluctuation résulte du fonctionnement en saturation en charge de la seconde galette 


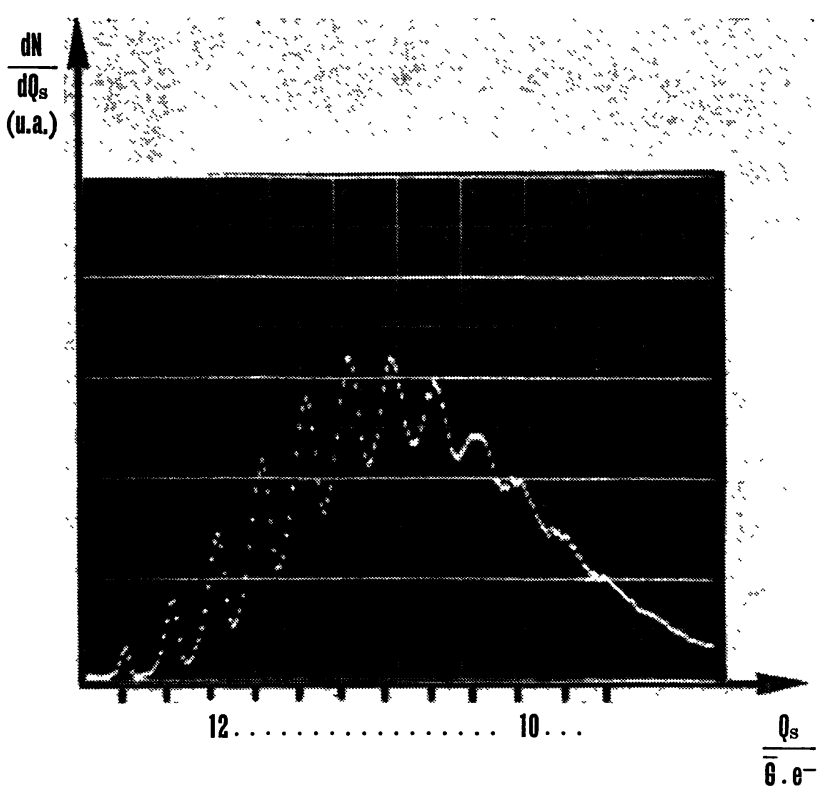

Fig. 8. - Spectre d'impulsions multiélectroniques obtenues à partir de l'émission d'un filament.

[Multielectron pulse spectrum obtained with a GMCC-GMCD association.]

dont la caractéristique de linéarité (Fig. 5 courbe b et Fig. 6) suit la loi empirique :

$$
G_{2}=G_{0_{2}}\left(\frac{q_{\mathrm{e}_{0}}}{q_{\mathrm{e}_{2}}}\right)^{\alpha} \text { avec } 0,7 \leqslant \alpha \leqslant 0,8
$$

La nature physique de $\alpha$ n'est pas très bien connue mais il y a tout lieu de penser que $\alpha$ est lié, lors du fonctionnement en saturation, à la modification des équipotentielles, donc du coefficient d'émission secondaire et des trajectoires électroniques dans les derniers paliers. Par suite, on a :

$$
\frac{d q_{\mathrm{s}_{2}}}{q_{\mathrm{s}_{2}}}=(1-\alpha) \frac{d q_{\mathrm{e}_{2}}}{q_{\mathrm{e}_{2}}} \# 0,3 \cdot \frac{d q_{\mathrm{e}_{2}}}{q_{\mathrm{e}_{2}}} .
$$

Quelle que soit la valeur de gain, la courbe de linéarité (Fig. 9) montre que la dynamique de détection,

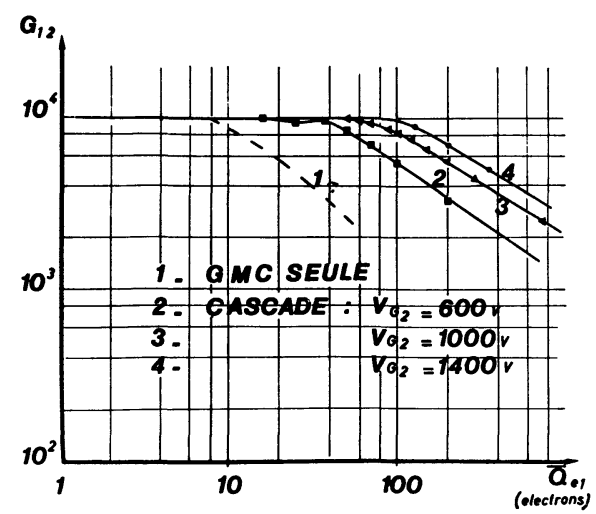

FIG. 9. - Influence de la tension de la seconde galette $\left(V_{\mathrm{G}_{2}}\right)$ sur la linéarité de détection de la cascade GMCC-GMCD.

[Influence of the 2 nd MCP voltage $\left(V_{\mathrm{G}_{2}}\right)$ on the linearity response of the GMCC-GMCD association.] $q_{\mathrm{e}_{1}}$, du multiplicateur à cascade de galettes est considérablement accrue. On vérifie bien que cet accroissement est directement proportionnel au champ électrique établi dans les canaux du second étage.

En régime de gain saturé, la linéarité est illustrée par la figure 8 où l'on peut séparer aisément des niveaux de détection $Q_{\mathrm{e}_{1}}$ correspondant à $n=1,2, \ldots$ 12 électrons dans le spectre d'impulsions multiélectroniques.

3.2.2 Dynamique de comptage. - En premier lieu proportionnelle au rapport courant de conduction/ charge délivrée par la galette, la dynamique de comptage se trouve accrue, dans ce montage, du fait des faibles valeurs de $d$ et $1 / d$ des canaux de la seconde GMC.

A gain élevé ( $\left.G \simeq 10^{7}, S / B>12\right)$, ce type d'association permet un fonctionnement à taux de comptage supérieur à celui admissible pour le premier étage à GMCC. La galette de sortie fonctionne alors en compensateur de gain en fournissant une charge quasi constante lorsque le gain de la première galette chute. Le principe de cette compensation est celui décrit pour la stabilisation des fluctuations de gain. Ainsi, une chute de $20 \%$ du niveau de sortie $q_{\mathrm{s}_{1}}$ de la GMCC n'entraîne qu'une variation de $4 \%$ du signal $Q_{\mathrm{s}_{2}}$, ce qui permet de porter la dynamique de comptage à $10^{6}-10^{7}$ impulsions. $\mathrm{s}^{-1} . \mathrm{cm}^{-2}$, soit un facteur $10 \mathrm{par}$ rapport à une galette à canaux courbes ou à un montage en chevron [8].

4. Applications. - De par ses propriétés uniques de détection liées à la nature des dynodes et à leurs caractéristiques géométriques, la GMC a trouvé un large domaine d'applications comme détecteur sans fenêtre, notamment sous la forme à 2 étages dite en chevron. Parmi ces applications, citons :

- la mesure de faibles flux continus de particules ou de photons (UV, X) par la méthode de comptage d'impulsions qui exige, entre autres, un détecteur présentant un bon rapport $S / B$ et pouvant fonctionner à haut taux de comptage ;

- la localisation à une ou deux dimensions avec une résolution spatiale élevée (imagerie par comptage) $[13,14]$;

- la mesure de temps à haute résolution temporelle, mesure qui exige un détecteur à gain élevé $\left(\geqslant 10^{7}\right)$ et à la fois une bonne résolution en amplitude et en temps.

Rappelons, pour ce dernier type d'application, que le multiplicateur à GMC offre des caractéristiques temporelles extrêmement intéressantes, notamment une fluctuation de temps de transit en électron unique $\left(\sigma_{\mathrm{T}} \simeq 50\right.$ à $\left.70 \mathrm{ps}\right)$ nettement inférieure ( 3 à 4 fois plus faible) à celle d'un multiplicateur conventionnel [15].

Parmi les applications mettant à profit à la fois ces caractéristiques élevées de résolution en amplitude et en temps, on peut citer, par exemple, les détecteurs de temps zéro utilisés dans les mesures par temps de vol 
pour l'identification de la masse des produits de réaction avec les ions lourds [16].

Ce type de multiplicateur trouve tout naturellement un grand intérêt pour la réalisation de PM plus performants, à savoir plus rapides (amélioration soit de la réponse impulsionnelle, soit de la résolution temporelle) et moins sensibles à un champ magnétique parasite (faible distance photocathode-entrée galette) ou de $\mathrm{PM}$ originaux tels que les $\mathrm{PM}$ à multianode [17, 18, 19].

En résumé, la structure particulière à 2 GMC décrite se présente comme une version améliorée du multiplicateur à chevron, améliorations qui portent directement ou indirectement sur :

- la résolution du $\mathrm{SEU}: \simeq 20 \%$ (4 à 5 fois plus faible) ;

- le taux de comptage (événements uniques) : qq $10^{6} \mathrm{~s}^{-1} \cdot \mathrm{cm}^{-2}$ (facteur $\geqslant 10$ );

- la dynamique de détection (facteur $\geqslant 10$ ) ;

- la résolution spatiale ;

- la résolution temporelle.

Les auteurs tiennent à remercier la Direction des Laboratoires d'Electronique et de Physique Appliquée qui a autorisé la publication de cet article, ainsi que MM. Loty pour ses discussions et critiques judicieuses et Ballanger pour l'aide efficace à la réalisation de ce travail.

Annexe. - EMPILEMENT DE PLUSIEURS ÉVÉNEMENTS A L'ENTRÉE DES MiCrocanauX D'UNE GMC. Plaçons-nous dans le cas limite où chaque microcanal ne peut détecter et multiplier qu'un seul événement primaire (électron ou photon unique).

En appelant $N$ le nombre de microcanaux disponibles, $n$ le nombre d'événements primaires répartis de façon homogène à l'entrée du multiplicateur, et en posant que les efficacités de collection et de détection sont égales à 1 , nous obtenons le schéma de fonctionnement suivant :

Pour le premier événement (aucun canal n'est occupé), le rendement de multiplication est $\eta_{1}=1$. Le deuxième a une probabilité $1 / N$ d'être pris en compte par le canal précédent. Son rendement de comptage est donc :

$$
\eta_{2}=1-\frac{1}{N}
$$

au troisième :

$$
\eta_{3}=1-\frac{2-\frac{1}{N}}{N}=1-\frac{2}{N}+\frac{1}{N^{2}}
$$

... au $\mathrm{i}^{\text {ième }}$ :

$$
\eta_{i}=1-\left(\begin{array}{l}
n \\
1
\end{array}\right) \frac{1}{N}+\left(\begin{array}{l}
n \\
2
\end{array}\right) \frac{1}{N^{2}}+\cdots+(-1)^{i}\left(\begin{array}{l}
n \\
i
\end{array}\right) \frac{1}{N^{2}}
$$

Pour une arrivée quasi simultanée de $n$ électrons (durée faible devant le temps de récupération), le nombre moyen d'électrons effectivement multiplié sera donc :

$$
\begin{aligned}
& \bar{n}^{\prime}=n-\frac{n(n-1)}{2 ! N}+\frac{n(n-1)(n-2)}{3 ! N^{2}} \cdots \\
& \bar{n}^{\prime}=-N\left[-\frac{n}{N}+\frac{n(n-1)}{2 ! N^{2}}-\frac{n(n-1)(n-2)}{3 ! N^{3}}+\cdots\right]
\end{aligned}
$$

soit

$$
\frac{\bar{n}^{\prime}}{n}=\frac{N}{n}\left[1-\left(1-\frac{1}{N}\right)^{n}\right]
$$

$N$ étant toujours très grand, nous pouvons écrire :

$$
\left.\left(1-\frac{1}{N}\right)^{n}=\exp \left[n \log \left(1-\frac{1}{N}\right)\right] \# \exp \left(-\frac{n}{N}\right)\right]
$$

d'où l'expression de $\bar{n}^{\prime} / n$, représentant le pourcentage d'événements comptés, sachant qu'un microcanal ne peut compter qu'un seul événement :

$$
\frac{\bar{n}^{\prime}}{n}=\frac{N}{n}\left[1-\exp \left(-\frac{n}{N}\right)\right] \text {. }
$$

\section{Bibliographie}

[1] Pietri, G., Proc. of the 1973 Intern. Conf. on Instrum. for High Energy Physics, Frascati (Italy), 586 (1973).

[2] Photomultipliers (Philips Application Book) 1970, H. Kater and L. J. Thompson (Editors).

[3] Acta Electronica 16 (1973).

[4] Chalmeton, V., Chevalier, P., Acta Electronica 14 (1971) 99.

[5] Bоuтот, J. P., 4e J. Optique Spatiale, Marseille (1973), 291.

[6] Hill, G. E., Adv. Electron. Electron Phys. 40A (1976) 153.

[7] Audier, M., Delmotte, J. C., Boutot, J. P., 5 J. Optique Spatiale, Marseille (1975), 33.

[8] Goodrich, G. W., US Patent no 3374380 (1968).

[9] Parkes, W., Gott, R., Nucl. Instrum. Meth. 95 (1971), 487.

[10] Colson, N. B., Mc Pherson, J., King, F. T., Rev. Sci. Instrum. 44 (1973) 1694.
[11] Parkes, W., Evans, K., Mathieson, E., Nucl. Instrum Meth. 121 (1974) 151.

[12] Guest, A. J., Acta Electronica 14 (1971) 79.

[13] Timothy, J. G., ByBeE, R. L., Rev. Sci. Instrum. 46 (1975) 1615.

Tiмотнy, J. G., ByвEe, R. L., Rev. Sci. Instrum. 48 (1977) 292.

[14] Lampton, M., Paresce, F., Rev. Sci. Instrum. 45 (1974) 1098.

[15] PIetri, G., IEEE Trans. Nucl. Sci. NS 24 (1977) 228.

[16] GiraRd, J., Bolore, M., à paraître dans Nucl. Instrum. Meth.

[17] Lo, C. C., Lecomte, P., Leskovar, B., IEEE Trans. Nucl. Sci. NS 24 (1977) 302.

[18] Brisson, J. C., CombY, G., B. I. S. T. no 198 (1974) 95.

[19] Boutot, J. P., Delmotte, J. C., L'Onde Electrique 56 (1976) 59. 